\title{
Disponibilidade de nitrogênio de fontes minerais e orgânicas aplicadas em um Argissolo cultivado com videira ${ }^{1}$
}

\author{
Felipe Lorensini ${ }^{2}$, Carlos Alberto Ceretta ${ }^{3}$, Gustavo Brunetto ${ }^{4}$,Jackson Berticelli Cerini ${ }^{5}$, \\ Cledimar Rogério Lourenzi ${ }^{2}$, Lessandro De Conti ${ }^{6}$, Tadeu Luis Tiecher ${ }^{6}$, Denis Eduardo Schapanski ${ }^{6}$
}

\begin{abstract}
RESUMO
As videiras em produção tendem a aproveitar pequena quantidade do $\mathrm{N}$, derivado do fertilizante mineral, mesmo cultivadas em solos com textura arenosa e baixo teor de matéria orgânica, o que confere a esses solos baixa capacidade de fornecimento de nitrogênio (N). O objetivo deste trabalho foi avaliar, por meio de incubação, a disponibilidade de nitrogênio $(\mathrm{N})$ em solo com histórico de cultivo com videiras e submetido à aplicação de fontes minerais e orgânicas de nutrientes. Foram coletadas amostras de um Argissolo Vermelho, em um vinhedo e em uma área sob campo natural, no município de Rosário do Sul (RS). Após o preparo do solo, foram montados os seguintes tratamentos: solo do campo nativo; solo do vinhedo; solo do vinhedo + composto orgânico; solo do vinhedo + ureia revestida com polímeros e solo do vinhedo + ureia. Os solos foram incubados por 141 dias, e aos 0, 38, 70, 102 e 141 dias de incubação, foram determinados os teores de $\mathrm{N}-\mathrm{NH}_{4}{ }^{+} \mathrm{e} \mathrm{N}-\mathrm{NO}_{3}$, e calculados os teores de $\mathrm{N}$ mineral, mineralização líquida, $\mathrm{N}$ mineralizado em relação ao total e o $\mathrm{N}$ mineral acumulado. No solo de vinhedo submetido à aplicação de ureia revestida com polímeros e de ureia, a maior mineralização de $\mathrm{N}$ aconteceu no período inicial. A liberação de $\mathrm{N}$ do composto orgânico tem um maior sincronismo com a necessidade da videira pelo nutriente. As reservas de $\mathrm{N}$ total potencialmente mineralizável do solo podem suprir a demanda da videira pelo $\mathrm{N}$.
\end{abstract}

Palavras-chave: composto orgânico, ureia revestida com polímero, Vitis vinífera L.

\section{ABSTRACT}

\section{Nitrogen availability of organic and mineral sources applied in Sandy Typic cultivated with vine}

The objective of this study was to evaluate the nitrogen $(\mathrm{N})$ availability in a soil with history of growing vines and submitted to application of mineral and organic sources of nutrients. In October 2010, samples of a Sandy Typic Hapludalf soil were collected in a vineyard and in a natural pasture soil, in the municipality of Rosario do Sul (RS). The treatments consisted of: natural pasture and vineyard soil as control; vineyard soil + organic compost; vineyard soil + polymer coated urea and vineyard soil + urea. The soils were incubated and subsamples were taken at 0, 38, 70, 102 and 141 days of incubation to determine $\mathrm{N}_{-} \mathrm{NH}_{4}{ }^{+}$and $\mathrm{N}-\mathrm{NO}_{3}{ }^{-}$contents. Concentrations of mineral $\mathrm{N}$, net mineralization, $\mathrm{N}$ mineralization in relation to the total $\mathrm{N}$ and mineral buildup were calculated. In vineyard soils submitted to application of the polymer coated urea and urea, most $\mathrm{N}$ mineralization occurred in the initial period. Nitrogen release from the organic compound has better synchronicity with the vine requirements. The soil reserves of total nitrogen potentially mineralized may supply the vine demand for $\mathrm{N}$.

Key words: organic compost, polymer coated urea, Vitis vinifera L.

\footnotetext{
Recebido para publicação em 03/08/2012 e aprovado em 09/08/2013.

'Parte da dissertação de mestrado do primeiro autor. Trabalho realizado com recursos da Capes e do CNPq.

2 Engenheiro-Agrônomo, Mestre. Departamento de Solos, Universidade Federal de Santa Maria, Avenida Roraima, 1000, Bairro Camobi, 97105-900, Santa Maria, Rio Grande do SuI, Brasil. felipe.lorensini@hotmail.com (autor para correspondencia) (Bolsista do CNPq.); crlourenzi@yahoo.com.br

${ }_{3}^{3}$ Engenheiro-Agrônomo, Doutor. Departamento de Solos, Universidade Federal de Santa Maria, Avenida Roraima, 1000, Bairro Camobi, 97105-900, Santa Maria, Rio Grande do SuI, Brasil. carlosceretta@ufsm.br (Bolsista em Produtividade do CNPq.).

${ }^{4}$ Engenheiro-Agrônomo, Doutor. Departamento de Engenharia Agrícola, Universidade Federal de Santa Maria, Avenida Roraima, 1000, Bairro Camobi, 97105-900, Santa Maria, Rio Grande do SuI, Brasil. brunetto.gustavo@gmail.com

${ }^{5}$ Engenheiro-Agrônomo, Mestre. Departamento de Solos, Universidade Federal de Santa Maria, Avenida Roraima, 1000, Bairro Camobi, 97105-900, Santa Maria, Rio Grande do SuI, Brasil. jabcerini@yahoo.com.br

${ }^{6}$ Graduandos em Agronomia. Departamento de Solos, Universidade Federal de Santa Maria, Avenida Roraima, 1000, Bairro Camobi, 97105-900, Santa Maria, Rio Grande do SuI, Brasil. lessandrodeconti@gmail.com; tadeu.t@hotmail.com; schapanski@hotmail.com (Bolsistas do CNPq.).
} 


\section{INTRODUÇÃO}

Os solos sob campo natural da região sudeste do Rio Grande do Sul (RS), a partir da década de 80, foram incorporados ao sistema de produção de uvas. Esses solos, na sua maioria, apresentam textura arenosa e baixo teor de matéria orgânica, o que lhes confere baixa capacidade de fornecimento de nitrogênio $(\mathrm{N})$ e, por isso, anualmente, as videiras são submetidas à aplicação de fontes minerais e orgânicas de N.

Porém, as videiras em produção tendem a aproveitar pequena quantidade do $\mathrm{N}$ derivado do fertilizante mineral (Brunetto et al., 2006; Brunetto, 2008). Isso tem sido atribuído, especialmente, mas não só, ao aumento da disponibilidade de $\mathrm{N}$ mineral na solução, em virtude da mineralização do $\mathrm{N}$ contido na matéria orgânica do solo, que pode suprir parte da demanda das plantas. Por outro lado, o baixo aproveitamento do $\mathrm{N}$ pode ocorrer por causa da rápida liberação do nutriente dos fertilizantes, como a ureia, o que contribui para diminuir o sincronismo entre a liberação e a sua absorção pelas plantas e potencializa a sua perda para o ambiente (Brunetto et al., 2009). Assim, o uso de fertilizantes orgânicos, como o composto orgânico (Bertran et al., 2004), bem como de fertilizantes nitrogenados revestidos com polímeros, como a ureia revestida (Almeida et al., 2005; Azevedo et al., 2009), pode ser uma estratégia mais adequada para promover a liberação gradual de N, o que tende a aumentar o seu aproveitamento pela videira, mas, por outro lado, a quantidade mineralizada pode ser inferior à demandada pela planta.

O composto orgânico, que pode ser obtido pela compostagem aeróbica de resíduos orgânicos (Bertran et al., 2004), quando aplicado na superfície do solo apresenta pequena área de contato com o solo, o que pode retardar a atividade da biomassa microbiana e, por consequência, a sua decomposição e liberação de nutrientes, como o N (Fares et al., 2005; Baldi et al., 2010). Por outro lado, os fertilizantes nitrogenados, como a ureia, podem ser revestidos com polímeros e, com isso, é criada uma barreira física ao redor do grânulo do fertilizante, que pode estimular a liberação mais lenta do N, em comparação com a dos fertilizantes minerais nitrogenados não revestidos (Morgan e Sinclair, 2009).

A taxa de mineralização de $\mathrm{N}$ de fontes minerais ou orgânicas de nutrientes em solos tem sido estimada, usando-se o método da incubação (Stanford e Smith, 1972; Camargo et al., 1997). A interpretação dos dados obtidos pode ser melhorada, utilizando-se modelos matemáticos que consideram um único reservatório de $\mathrm{N}$ mineralizável no solo (Stanford e Smith, 1972) e que possibilitam determinar a fração do $\mathrm{N}$ orgânico $\left(\mathrm{N}_{\mathrm{org}}\right)$ potencialmente mineralizável e a constante de mineralização (Camargo et al., 1997). O objetivo deste trabalho foi avaliar a disponibilidade de $\mathrm{N}$ no solo com histórico de cultivo com videiras e submetido à aplicação de fontes minerais e orgânicas de nutrientes.

\section{MATERIAL E MÉTODOS}

O trabalho foi realizado no Laboratório de Química e Fertilidade do Solo da Universidade Federal de Santa Maria (UFSM), em Santa Maria, RS, Brasil. Em outubro de 2010, foram coletadas amostras de um Argissolo Vermelho (Santos et al., 2006), na camada de 0-20 cm, em uma área cultivada, durante oito anos, com videira, e em uma área, adjacente ao vinhedo, sob campo nativo, sem histórico de cultivo e adubação. O vinhedo era composto pelo cultivar Cabernet Sauvignon, enxertado sobre porta-enxerto SO4, com densidade de 3.704 plantas por hectare (1,0 m entre plantas x 2,7 m entre linhas), conduzido em sistema de espaldeira e instalado em uma propriedade vitivinícola, no município de Rosário do Sul, RS, em $30^{\circ}$ $15^{\prime} 26.75^{\prime \prime}$ S, e $54^{\circ} 58^{\prime} 43.72^{\prime \prime}$ W. O clima da região é subtropical úmido, tipo Cfa 2 (Kottek et al., 2006), com temperatura média anual de $17,5^{\circ} \mathrm{C}$ e precipitação média anual de $1450 \mathrm{~mm}$.

O solo foi coletado, no vinhedo, ao longo das linhas de plantio das videiras e, aleatoriamente, no campo nativo e, em seguida, eles foram armazenados. Posteriormente, foram passados em peneira com malha de $4 \mathrm{~mm}$ e foi corrigida a umidade para $80 \%$ da capacidade de campo. Logo depois, 157,5 g de solo foram colocados em recipientes de acrílico com capacidade de $110 \mathrm{~mL}$, que correspondem às unidades experimentais, e submetido a duas compactações (para maior uniformização da densidade), resultando o volume final de $100 \mathrm{~mL}$ e, com isso, obteve- se a densidade de $1,57 \mathrm{~g} \mathrm{~cm}^{-3}$, semelhante à encontrada no solo do vinhedo e do campo nativo.

Os tratamentos foram: solo do campo nativo, solo do vinhedo, solo do vinhedo + composto orgânico, solo do vinhedo + ureia revestida com polímeros e solo do vinhedo + ureia.

O composto orgânico apresentou as seguintes características em base seca: matéria seca de 84,1\%; $\mathrm{pH}$ em água 9,6; $\mathrm{N}$ na forma de amônio $\left(\mathrm{N}_{-} \mathrm{NH}_{4}^{+}\right)$0,46\%; $\mathrm{N}$ na forma de nitrato $\left.\left(\mathrm{N}^{-\mathrm{NO}_{3}}\right)^{-}\right) 0,40 \%$; $\mathrm{N}$ total $\left(\mathrm{N}_{\text {tot }}\right) 1,9 \%$; P total $1,73 \%$; $\mathrm{K}$ total $3,2 \%$; Ca total $2,5 \%$; Mg total $0,2 \%$ e carbono orgânico total de $19,9 \%$. A ureia revestida com polímero apresentava $41,33 \%$ de $\mathrm{N}_{\text {tot }}$ e, a ureia sem revestimento, $45 \%$ de N. A quantidade de cada fonte de N, adicionada ao solo em cada frasco de acrílico, correspondeu a $40 \mathrm{~kg}$ de $\mathrm{N}$ total ha ${ }^{-1}$, equivalente a $1224,3 \mathrm{mg}$ de composto; 51,3 $\mathrm{mg}$ de ureia revestida com polímero e 47,10 mg de ureia.

Os fertilizantes foram adicionados à superfície do solo, simulando a aplicação superficial de fontes de N, 
normalmente realizada nos solos cultivados com videira. Em seguida, quatro frascos de acrílico, que representaram as quatro repetições de cada tratamento, foram acondicionados em frascos de $1500 \mathrm{~mL}$, para evitar a perda excessiva de umidade e, logo depois, foram armazenados em câmara de incubação, com temperatura constante de $25 \pm 1^{\circ} \mathrm{C}$. O delineamento experimental usado foi inteiramente casualizado, com quatro repetições. A cada três dias, os recipientes com as unidades experimentais foram abertos, por 15 minutos, em local arejado, para evitar a saturação de gases no interior dos recipientes. A umidade de cada unidade experimental foi monitorada diariamente e, quando necessário, foi adicionada água destilada, para manter a capacidade de campo a $80 \%$.

Aos 0, 38, 70, 102 e 141 dias depois do início da incubação, equivalente aos seguintes períodos fenológicos da videira: brotação, florescimento, grão tamanho chumbinho, mudança de cor e colheita da uva, respectivamente, o solo foi retirado dos frascos, homogeneizado e separado em duas porções. Logo depois, uma porção foi submetida à análise de $\mathrm{N}-\mathrm{NH}_{4}^{+}{ }^{+} \mathrm{N}-\mathrm{NO}_{3}^{-}$, seguindo-se a metodologia proposta por Tedesco et al. (1995). A segunda porção foi utilizada para a determinação de umidade (Tedesco et al., 1995) e, com isso, os valores de N$\mathrm{NH}_{4}{ }^{+} \mathrm{e} \mathrm{N}-\mathrm{NO}_{3}{ }^{-}$obtidos foram expressos em mg de $\mathrm{N} \mathrm{kg}^{-1} \mathrm{de}$ solo seco.

Tabela 1. Atributos do solo Argissolo Vermelho sob campo natural e cultivado com videira

\begin{tabular}{|c|c|c|}
\hline Atributos & Campo nativo & Vinhedo \\
\hline Argila, $\mathrm{g} \mathrm{kg}^{-1(1)}$ & 71 & 94 \\
\hline Silte, $\mathrm{g} \mathrm{kg}^{-1(1)}$ & 104 & 251 \\
\hline Areia, $\mathrm{g} \mathrm{kg}^{-1(1)}$ & 825 & 655 \\
\hline Matéria orgânica, $\mathrm{g} \mathrm{kg}^{-1(2)}$ & 13,0 & 12,0 \\
\hline $\mathrm{pH}$ em $\mathrm{H}_{2} \mathrm{O}^{(3)}$ & 4,4 & 6,4 \\
\hline Índice SMP ${ }^{(3)}$ & 6,4 & 7,1 \\
\hline $\mathrm{N}-\mathrm{NH}_{4}^{+}, \mathrm{mg} \mathrm{kg}^{-1}(3)$ & 1,5 & 0,8 \\
\hline $\mathrm{N}-\mathrm{NO}_{3}^{-}, \mathrm{mg} \mathrm{kg}^{-1(3)}$ & 9,3 & 9,4 \\
\hline $\mathrm{N}$ total, $\mathrm{mg} \mathrm{kg}^{-1(3)}$ & 613 & 508 \\
\hline $\mathrm{P}$ disponível, $\mathrm{mg} \mathrm{dm}^{-3(4)}$ & 3,8 & 96,4 \\
\hline $\mathrm{K}$ trocável, $\mathrm{mg} \mathrm{dm}^{-3(4)}$ & 44,0 & 82,0 \\
\hline Ca trocável, $\mathrm{cmol}_{\mathrm{c}} \mathrm{dm}^{-3}(5)$ & 0,4 & 1,0 \\
\hline Mg trocável, $\mathrm{cmol}_{\mathrm{c}} \mathrm{dm}^{-3(5)}$ & 0,2 & 0,5 \\
\hline Al trocável, $\mathrm{cmol}_{\mathrm{c}} \mathrm{dm}^{-3}(5)$ & 0,4 & 0,0 \\
\hline $\mathrm{H}+\mathrm{Al}, \mathrm{cmol}_{\mathrm{c}} \mathrm{dm}^{-3}(3)$ & 2,9 & 1,2 \\
\hline $\mathrm{CTC}_{\mathrm{pH} 7,0}, \mathrm{cmol}_{\mathrm{c}} \mathrm{dm}^{-3}(6)$ & 3,6 & 2,9 \\
\hline $\mathrm{CTC}_{\text {efetiva }}, \mathrm{cmol}_{\mathrm{c}} \mathrm{dm}^{-3}(6)$ & 1,1 & 1,7 \\
\hline Saturação por bases, \% ${ }^{(3)}$ & 19,7 & 58,8 \\
\hline Saturação por alumínio, \% ${ }^{(3)}$ & 34,4 & 0,0 \\
\hline \multicolumn{3}{|c|}{ 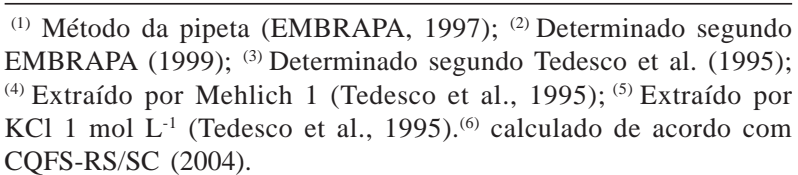 } \\
\hline
\end{tabular}

$\mathrm{O} \mathrm{N}$ na forma de nitrito $\left(\mathrm{N}-\mathrm{NO}_{2}^{-}\right)$, nas amostras de solos foi desprezado, porque seus teores foram muito pequenos nos solos estudados. Com os teores de $\mathrm{N}_{-} \mathrm{NH}_{4}{ }^{+} \mathrm{e}$ $\mathrm{N}-\mathrm{NO}_{3}$ - foram obtidos os valores de $\mathrm{N}$ mineral $\left(N_{\text {min }}\right)$ (equação 1):

$\mathrm{N}_{\text {min }}=\mathrm{N}-\mathrm{NH}_{4}^{+}+\mathrm{N}-\mathrm{NO}_{3}^{-}$

Equação 1

A evolução da taxa de mineralização de $\mathrm{N}$ dos diferentes tratamentos ao longo da incubação foi avaliada por meio da mineralização liquida $\left(\mathrm{N}_{\mathrm{liq}}\right)$ (equação 2):

$N_{\text {liq }}=N_{\text {min }}$ solocomfertilizante $-N_{\min }$ do solo testemunha Equação 2 em que: $N_{\text {liq }}=$ mineralização líquida $\left(\mathrm{mg} \mathrm{de}^{\mathrm{N} \mathrm{kg}}{ }^{-1}\right) ; N_{\min }=$ teores de $\mathrm{N}-\mathrm{NH}_{4}{ }^{+}+\mathrm{N}^{-} \mathrm{NO}_{3}^{-}$em cada tempo avaliado (mg de $\mathrm{N} \mathrm{kg}^{-1}$ ).

A $N_{l i q}$ indica se houve predomínio da mineralização do $\mathrm{N}$ (valor positivo), ou da imobilização do $\mathrm{N}$ (valor negativo), a cada tempo de coleta e para cada combinação solomaterial orgânico (Giacomini, 2005). Também foi calculado o $\% N_{\min }$ sobre o $\mathrm{N}$ total $\left(N_{\text {tot }}\right)$ (equação 3$)$ :

$\% N_{\min }=N_{\min } \times 100 / N_{t o t}$ Equação 3

em que: $\% N_{\min }=$ percentagem de $\mathrm{N}$ que foi mineralizado em relação ao $N_{\text {tot }}(\%) ; N_{\text {min }}=$ teores de $\mathrm{N}_{-} \mathrm{NH}_{4}{ }^{+}+\mathrm{N}_{-} \mathrm{NO}_{3}{ }^{-}$ em cada tempo avaliado $\left(\mathrm{mg} \mathrm{de} \mathrm{N} \mathrm{kg}^{-1}\right) ; N_{\text {tot }}=\mathrm{N}$ adicionada por via do fertilizante, mais o $\mathrm{N}$ nativo (orgânico + mineral) do solo, determinado segundo Tedesco et al.



A cinética de mineralização, os potenciais de mineralização (No) e a constante de mineralização $(k)$ de $\mathrm{N}$ foram obtidos a partir dos valores acumulados de $N_{\text {min }}$ durante o período de incubação e ajustados pelo modelo matemático de progressão não linear. O modelo empregado foi o proposto por Stanford e Smith (1972), que considera que apenas uma fração do $N_{\text {org }}$ do solo é potencialmente mineralizável e que o processo de mineralização segue uma cinética de primeira ordem, sendo definido pela equação exponencial simples (equação 4):

$N m=N o\left(1-e^{-k t}\right)$

Equação 4

em que: $\mathrm{Nm}=\mathrm{N}$ mineralizado acumulado em um período de tempo $\left(\mathrm{mg}\right.$ de $\left.\mathrm{N} \mathrm{kg}^{-1}\right) ; N o=$ potencial de mineralização do $\mathrm{N}\left(\mathrm{mg} \mathrm{de}^{\mathrm{N} \mathrm{kg}} \mathrm{kg}^{-1}\right) ; k=$ constante de mineralização $\left(\operatorname{dia}^{-1}\right)$; e $\mathrm{t}=\mathrm{o}$ tempo (dias).

A redução do $\mathrm{No}$, função da mineralização $(\mathrm{No}-\mathrm{Nm})$, em cada evento, foi determinada com os dados ajustados pelo modelo proposto por Stanford e Smith (1972). Para os dados relativos aos teores de $\mathrm{N}-\mathrm{NH}_{4}^{+}$e $\mathrm{N}-\mathrm{NO}_{3}{ }^{-}$ foi calculado o erro padrão da média, em cada data de avaliação. 


\section{RESULTADOS E DISCUSSÃO}

O solo do campo nativo, a partir de 70 dias de incubação, apresentou teores de $\mathrm{N}-\mathrm{NH}_{4}{ }^{+}$maiores que os obtidos nos solos do vinhedo, sem a aplicação de fonte de $\mathrm{N}$ e com a aplicação de composto orgânico, ureia revestida com polímeros e ureia (Figura 1a). Isso pode ser atribuído à mineralização de compostos orgânicos, presentes em maior quantidade no solo do campo nativo. No solo do vinhedo + ureia revestida e solo do vinhedo + ureia, foram encontrados os maiores teores de $\mathrm{N}_{-} \mathrm{NH}_{4}^{+}$, no início da incubação (tempo zero), e aos 38 dias após o início da incubação. Esse comportamento era esperado e ocorreu por causa da rápida solubilização das duas fontes de $\mathrm{N}$ mineral. Por outro lado, os teores de $\mathrm{N}_{-} \mathrm{NH}_{4}^{+}$, no solo do vinhedo + composto orgânico, no tempo zero da incubação e aos 38 dias após seu início, foram menores que os encontrados nos tratamentos solo do vinhedo + ureia revestida e solo do vinhedo + ureia. Isso pode ser explicado pela aplicação dos fertilizantes na superfície do solo, o que diminui a área de contato solo-fertilizante orgânico e, por consequência, retarda a atividade da biomassa microbiana. Soma-se a isso o fato de que, do total de $\mathrm{N}$ adicionado por via do composto, apenas 45,3\% está disponível, enquanto o $\mathrm{N}$ adicionado por via da ureia revestida e da ureia está $100 \%$ na forma disponível.

Os maiores teores de $\mathrm{N}_{-} \mathrm{NO}_{3}^{-}$, aos 38, 70102 e 141 dias após o início da incubação, foram encontrados no solo do vinhedo + ureia revestida e no solo do vinhedo + ureia, por causa da rápida solubilização dos fertilizantes, que liberam $\mathrm{N}_{-} \mathrm{NH}_{4}^{+}$, que é oxidado à $\mathrm{N}^{-\mathrm{NO}_{2}}$ - pelas bactérias do gênero nitrobacter e, posteriormente, a N-NO ${ }_{3}^{-}$, por nitrossomonas (Cantarella et al., 2007) (Figura 1b). Com isso, pode-se inferir que, em solos de textura arenosa, submetidos à aplicação de ureia e de ureia revestida, a

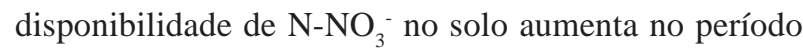
compreendido entre a adição dos fertilizantes, que, neste trabalho, simulou a brotação da videira, e aos 38 dias após sua adição, que correspondeu ao florescimento. Isso indica que, caso fossem utilizadas essas fontes de $\mathrm{N}$ e aplicado o total, ou mesmo metade, da dose do nutriente, como proposto pela Comissão de Química e Fertilidade do Solo (CQFS-RS/SC) (Sociedade Brasileira de Ciência do Solo, 2004), durante o início da brotação da videira, parte do $\mathrm{N}$ aplicado pode ser lixiviado na forma de $\mathrm{N}_{-} \mathrm{NO}_{3}$ uma vez que a videira apresenta a maior absorção de $\mathrm{N}$ durante o período de florescimento, especialmente porque, neste estádio fenológico, acontece a emissão de um grande número de raízes jovens, finas e que são responsáveis pela maior absorção de água e nutrientes, entre eles, o N (Vos et al, 2004; Schreiner e Scagel, 2006); coincidindo com os 38 dias depois da aplicação do N. Em todas as épocas de coleta do solo incubado, os teores de
$\mathrm{N}-\mathrm{NO}_{3}{ }_{3}$ no solo do campo nativo, no solo do vinhedo e no solo do vinhedo + composto orgânico foram similares e menores que os encontrados nos tratamentos solo do vinhedo + ureia revestida e solo do vinhedo + ureia. Os menores teores de $\mathrm{N}_{-} \mathrm{NO}_{3}{ }^{-}$no solo do vinhedo + composto orgânico, em comparação com os do solo do vinhedo + ureia revestida e solo de vinhedo + ureia, podem ser atribuídos à baixa taxa de liberação do $\mathrm{N}$ contido no composto orgânico.

Em todos os períodos avaliados, no solo do vinhedo submetido à aplicação de composto orgânico, ureia e ureia revestida, os valores de mineralização líquida $\left(N_{l i q}\right)$ foram positivos (Figura 1c), indicando que houve predomínio do processo de mineralização sobre o processo de imobilização (Giacomini, 2005). Isso ocorreu porque o teor de matéria orgânica natural do solo do vinhedo era $12 \mathrm{~g} \mathrm{~kg}^{-1}$, interpretado como baixo $\left(<25 \mathrm{~g} \mathrm{~kg}^{-1}\right.$ ) (Sociedade Brasileira de Ciência do Solo, 2004) e apresenta uma relação C/N inferior a 10 , sendo que abaixo de 20 predomina a mineralização. Os valores de $N_{l i q}$ nos solos do vinhedo + ureia e no solo do vinhedo + ureia revestida foram similares em todas as épocas de coleta, a partir de 38 dias após o início da incubação, e maiores que os obtidos no solo do vinhedo + composto orgânico, provavelmente, por causa dos maiores teores de $N_{\min }$ (Figura 1e).

Os resultados de percentagem de $\mathrm{N}$ mineralizado em relação ao $\mathrm{N}_{\text {tot }}\left(\% N_{\text {min }}\right)$ mostram que, aproximadamente, $8 \%$ do $\mathrm{N}_{\text {tot }}$ do solo do campo nativo e do solo do vinhedo sem a aplicação de fonte de $\mathrm{N}$ foi mineralizado aos 141 dias, comportamento similar ao encontrado no solo do vinhedo + composto orgânico (Figura 1d). Por sua vez, no solo do vinhedo que recebeu a adição de ureia e de ureia revestida, também aos 141 dias de incubação, os valores de $\% N_{\text {min }}$ encontrados situaram-se em torno de $24 \%$. Esse valor é maior que o encontrado nos solos de campo nativo e de vinhedo sem a adição de fertilizantes ( $8 \%$ do $\mathrm{N}_{\text {tot }}$ ), porque ocorreu a mineralização do $\mathrm{N}$ contido no próprio fertilizante, o que fica evidenciado quando se subtrai o $\mathrm{N}_{\text {liq }}$ do $\mathrm{N}_{\min }$ acumulado (Figura 1c e 1e).

$\mathrm{O} \mathrm{N}_{\min }$ acumulado dos tratamentos solo do vinhedo + ureia revestida e do solo do vinhedo + ureia, incubados durante 141 dias, foi exponencial e a queda na inclinação da curva, aos 38 dias, foi abrupta (Figura 1e), sendo que a partir de 38 dias de incubação o processo atingiu valores mais estáveis, com menores acréscimos da quantidade de $\mathrm{N}_{\text {min }}$ em função do tempo. Resultados semelhantes foram observados por Camargo et al. (1997), que, em dez tipos de solos do RS, encontraram maiores quantidades de $\mathrm{N}_{\text {min }}$ até 28 dias depois do início da incubação. Por outro lado, o $\mathrm{N}_{\text {min }}$ acumulado do solo do campo nativo, do solo do vinhedo e do solo do vinhedo + composto orgânico foi linear ao longo dos 141 dias de incubação. Aos 38 dias de incubação, nos tratamentos em que foi utilizada ureia 



E



líquida (c); $\mathrm{N}$ mineralizado em relação ao total (d); $\mathrm{N}$ mineralizado acumulado (e), curvas de $\mathrm{N}$ mineralizado acumulado (f) e variação líquida de $\mathrm{Nm}$ e quantidade de $\mathrm{N}$ potencialmente mineralizável disponível à mineralização (No - Nm), ajustadas pelo modelo de Stanford \& Smith (1972) (g), durante a incubação de solos submetidos à aplicação de fontes de nitrogênio. 
revestida e ureia, observou-se que, respectivamente, do total de $\mathrm{N}_{\text {min }}, 94$ e $89 \%$ ocorreram até 38 dias da incubação. Por outro lado, no campo nativo, solo do vinhedo e solo do vinhedo + composto orgânico, respectivamente, 45, 63 e $64 \%$, haviam sido encontrados até 38 dias da incubação.

O modelo exponencial simples proposto por Stanford e Smith (1972) (Figura 1f) ajustou adequadamente os resultados de $\mathrm{Nm}$, em geral, similares aos de $N_{\text {min }}$ no tempo zero e aos 38, 70, 102 e 141 dias, e estimou os valores de No próximos aos de $N_{\text {min }}$ aos 141 dias (Figura 1e). Esses resultados concordam com os obtidos por Camargo et al. (1997), que, em 10 solos representativos do RS e incubados durante 224 dias sem aplicação de $\mathrm{N}$, relatam que o modelo estimou adequadamente os valores de $\mathrm{No}$, com os valores de No ligeiramente inferiores aos de $N_{\min }$ acumulado aos 224 dias. Da mesma forma, Camargo et al. (2002) relatam que os modelos exponenciais simples, como o proposto por Stanford e Smith (1972), descrevem adequadamente a mineralização do $N_{o r g}$ nos solos.

$\mathrm{O}$ valor de $\mathrm{No}$, maior no solo do campo nativo $(67,1 \mathrm{mg}$ de $\mathrm{N} \mathrm{kg}^{-1}$ ), que o do solo do vinhedo (35,7 $\mathrm{mg}_{\text {de }} \mathrm{N} \mathrm{kg}^{-1}$ ), pode ser atribuído ao seu maior teor de $N_{\text {tot }}$ (Figura 1f). O solo do vinhedo + composto orgânico apresentou No de

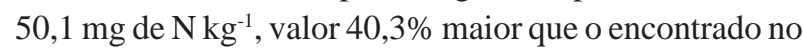
solo do vinhedo sem a aplicação de fonte de $\mathrm{N}(35,7 \mathrm{mg}$ de $\left.\mathrm{N} \mathrm{kg}^{-1}\right)$. Entretanto, nos solos do vinhedo com a adição de ureia e de ureia revestida, os valores de $\mathrm{No}$ foram de,

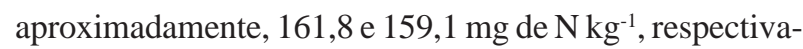
mente. Esses resultados demonstram que cerca de 7,0\% do reservatório de $\mathrm{N}_{\text {tot }}$ do solo do campo nativo e do solo do vinhedo é potencialmente mineralizado, no período entre a brotação (tempo zero) e colheita da uva (141 dias depois do início da incubação). O solo do campo nativo, do vinhedo sem a aplicação de fontes de $\mathrm{N}$, do vinhedo + composto orgânico, do vinhedo + ureia revestida e do vinhedo + ureia apresentaram $k$ ( constante de mineralização em dias) de 0,0114; 0,0233; 0,0265; 0,0879 e 0,0599, respectivamente (Figura 1f). Os maiores valores de $k$ nos solos do vinhedo + ureia revestida e ureia podem ser atribuídos à maior solubilidade do $\mathrm{N}$, enquanto a variação dos valores de $k$ entre os dois solos sugere que os solos podem diferir não somente com relação à fração de $\mathrm{N}_{\text {org }}$, potencialmente mineralizado, mas também em relação à atividade microbiana (Camargo et al., 2002).

Considerando-se que os solos do campo nativo e do vinhedo sem a aplicação de fontes de $\mathrm{N}$ apresentaram valores de $\mathrm{No}$ de 67,1 e 35,1 mg de $\mathrm{N} \mathrm{kg}^{-1}$, respectivamente, e que foram coletados na camada de $0-20 \mathrm{~cm}$ e apresentaram densidade de $1,575 \mathrm{~g} \mathrm{~cm}^{-3}(3.150 .000 \mathrm{~kg}$ de solo ha-1), pode-

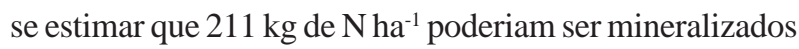
no solo do campo nativo e $110,5 \mathrm{~kg}$ de $\mathrm{N}^{-1}$ no solo do vinhedo, ao longo de 141 dias de cultivo da videira. Segundo Brunetto (2008), as videiras Cabernet Sauvignon, culti- vadas em solo Argissolo, na Campanha Gaúcha do RS, acumulam 24,5 g de $\mathrm{N}$ total planta ${ }^{-1}$, desconsiderando-se a quantidade de $\mathrm{N}$ acumulada nas raízes. Com isso, esperase que, no vinhedo, onde foi coletado o solo para a realização deste trabalho, que possui 3704 plantas ha-1 ${ }^{-1}$ o acúmulo total de $\mathrm{N}$ seja de $90,7 \mathrm{~kg}$ de $\mathrm{N} \mathrm{ha}^{-1}$, quantidade menor que a mineralizada no solo. Isso pode ser uma das possíveis explicações para o pequeno aproveitamento de $\mathrm{N}$ da ureia enriquecida com isótopos de ${ }^{15} \mathrm{~N}$, aplicada em videiras Cabernet Sauvignon, cultivadas em solo com textura arenosa, na Campanha Gaúcha do RS, mas, também, em solo com textura média, da região da Serra Gaúcha do RS (Brunetto, 2008). Para atender a uma demanda de absorção

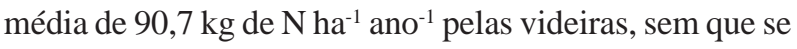
considerem as perdas do $\mathrm{N}_{\text {min }}$, seria suficiente uma



A variação líquida de $\mathrm{Nm}$ e a quantidade de $N o$ disponível à mineralização (No - Nm), obtida pelo modelo exponencial simples proposto por Stanford e Smith (1972), é apresentada na Figura 1g. Verifica-se que, no solo do campo nativo, do vinhedo sem a aplicação de fontes de $\mathrm{N}$ e do vinhedo + composto orgânico, durante 141 dias, a redução do $N o$, em função da mineralização (No - Nm), é gradual. Embora apenas 10,2\% do $\mathrm{N}$ adicionado por via do composto tenham sido mineralizados, durante os 141 dias de incubação, o que é equivalente a $4,0 \mathrm{~kg}$ de $\mathrm{N} \mathrm{ha}^{-1}$, em comparação com o mesmo parâmetro do solo do vinhedo que recebeu a adição de ureia revestida e de ureia, que mineralizou $85 \%$ do $\mathrm{N}$, adicionado por via dos fertilizantes, o que é equivalente a $34 \mathrm{~kg}$ de $\mathrm{N} \mathrm{ha}^{-1}$, a mineralização gradual do $\mathrm{N}$ contido no composto impede que o $\mathrm{N}$ mineralizado seja transferido para fora do sistema, principalmente por lixiviação de $\mathrm{NO}_{3}^{-}$, bem como melhora o sincronismo entre a liberação do N e a absorção pela videira, ao longo do tempo. Além disso, a adição de composto orgânico repõe as reservas de $\mathrm{N}$ do solo, impedindo uma depleção em função da mineralização.

\section{CONCLUSÕES}

Nos solos de vinhedo que receberam ureia revestida com polímeros e ureia, aplicadas na superfície do solo, a maior mineralização de $\mathrm{N}$ aconteceu no período inicial, até os 38 dias de incubação.

A mineralização de $\mathrm{N}$ do composto orgânico aplicado no solo do vinhedo apresenta maior sincronismo com a necessidade da videira pelo nutriente, quando comparado com a do $\mathrm{N}$ da ureia revestida e com a da ureia. Por isso, o composto orgânico pode ser indicado como uma boa fonte de $\mathrm{N}$ para a videira.

As reservas de $\mathrm{N}$ total potencialmente mineralizável, presentes no solo do campo nativo e no solo do vinhedo, podem suprir a necessidade de $\mathrm{N}$ pela videira. 


\section{AGRADECIMENTOS}

À Capes, à Fapergs e ao CNPq, pelo financiamento e pelas bolsas concedidas.

\section{REFERÊNCIAS}

Almeida TRP, Leonel S, Tecchio MA \& Mischan MM (2005) Formação do pomar de tangerina 'Poncã', em função da adubação química e orgânica. Revista Brasileira de Fruticultura, 27:288-291.

Azevedo EB, Marinho CS, Muniz RA \& Carvalho AJC (2009) Substratos fertilizados com uréia revestida e o crescimento e estado nutricional da muda de citros. Acta Scientiarum. Agronomy, 31:129-137.

Baldi E, Toselli M, Eissenstat DM \& Marangoni B (2010) Organic fertilization leads to increased peach root production and lifespan. Tree Physiology, 0:1-10.

Bertran E, Sort X, Soliva M \& Trillas I (2004) Composting winery waste: sludges and grape stalks. Bioresource technology, 95:203208.

Brunetto G, Kaminski J, Melo GWB, Bruning FS \& Mallmann F (2006) Destino do nitrogênio em videiras 'Chardonnay' e 'Riesilng Renano' quando aplicado no inchamento das gemas. Revista Brasileira de Fruticultura, 28:497-500.

Brunetto G (2008) Nitrogênio em videira: Recuperação, acumulação e alterações na produtividade e na composição da uva. Tese Doutorado. Universidade Federal de Santa Maria, Santa Maria. 139 p.

Brunetto G, Ceretta CA, Kaminski J, Melo GW, Girotto E, Trentin EE, Lourenzi CR, Renan RCB \& Gatiboni LC (2009) Produção e composição química da uva de videiras Cabernet Sauvignon submetidas à adubação nitrogenada. Ciência Rural, 39:20352041 .

Camargo FAO, Gianello C \& Vidor C (1997) Potencial de mineralização de nitrogênio em solos do Rio Grande do Sul. Revista Brasileira de Ciência do Solo, 21:575-579.

Camargo FAO, Gianello C, Tedesco MJ, Riboldi J, Meurer EJ \& Bissani CA (2002) Empirical models to predict soil nitrogen mineralization. Ciência Rural, 12:393-399.

Cantarella H, Novais RF, Alvarez VH, Barros NF, Fontes RLF, Cantarutti RB \& Neves JCL (2007) Nitrogênio. In Novais RF, Alvarez VH, Barros NF, Fontes RLF, Cantarutti, RB \& Neves JCL. (Eds.). Fertilidade do Solo $2^{\mathrm{a}}$ ed. Viçosa, Sociedade Brasileira de Ciência do Solo. p.375-470.

Fares F, Albalkhi A, Dec J, Bruns MA \& Bollag JM (2005) Physicochemical characteristics of animal and municipal wastes decomposed in arid soils. Journal of Environmental Quality, 34:1392-1403.

Giacomini, SJ (2005) A avaliação e modelização da dinâmica de carbono e nitrogênio em solo com o uso de dejetos de suínos. Tese Doutorado. Universidade Federal de Santa Maria, Santa Maria. 247p.

Kottek M, Grieser J, Beck C, Rudolf B \& Rubel F (2006) World map of the Köppen-Geiger climate classification updated. Meteorologische, 15:259-263.

Morgan KT \& Sinclair TR (2009) Release mechanisms for solwand controlled-release fertilizers and strategies for their use in vegetable production. Hortechnology, 19:10-12.

Santos HG, Jacomine PKT, Anjos LHC, Oliveira VA; Oliveira JB, Coelho MR, Lumbreras JF \& Cunha TJF (2006) Sistema brasileiro de classificação de solos. Rio de Janeiro, Embrapa Solos. 412p.
Schreiner RP \& Scagel CF (2006) Nutrient uptake and distribution in a mature 'Pinot Noir' vineyard. HortScience, 41:336-345.

Sociedade Brasileira de Ciência do Solo (2004) Núcleo Regional Sul. Comissão de Química e Fertilidade do Solo RS/SC. Manual de adubação e de calagem para os Estados do Rio Grande do Sul e Santa Catarina. $10^{\mathrm{a}}$ ed. Porto Alegre, SBCS. 400p.

Stanford G \& Smith SJ (1972) Nitrogen mineralization potentials of soils. Soil Science Society of America Journal, 36: 465-472.

Tedesco, MJ (1995) Análises de solos, plantas e outros materiais. $2^{\mathrm{a}}$ ed. Porto Alegre, UFRGS. 174p. (Boletim Técnico, 5).

Vos RJ, Zabadal, TJ \& Hanson EJ (2004) Effect of nitrogen application timing on $\mathrm{N}$ uptake by Vitis labrusca in a shortseason region. American Journal of Enology and Viticulture, $55: 246-252$. 